\title{
Consumo de tabaco entre los mexicanos y sus descendientes, en Estados Unidos de América
}

\author{
Ralph S C araballo, MPH, PhD, (1) Chung-W on Lee, MA, Ph D.. ${ }^{(2)}$
}

\section{Caraballo RS, Lee CW. \\ Consumo de tabaco entre los mexicanos y sus descendientes, en Estados Unidos de América. Salud Publica Mex 2004;46:241-250. \\ El texto completo en inglés de este artículo está disponible en: http://www.insp.mx/salud/index.html}

\section{Resumen}

Objetivo. Presentar información obtenida en encuestas y estudios en Estados Unidos de América sobre el hábito de fumar cigarillos, o el uso de cualquier otro producto que contenga tabaco, entre los mexicanos que viven en ese país. Material y métodos. Se utilizaron diferentes encuestas 0 sistemas de información. Cada encuesta 0 sistema de información tiene su propia metodología y manera de recoger los datos. Las encuestas o sistemas de información utilizados en este informe fueron: la Youth Risk Behavior Survey, 1991-2001, la N ational Survey on Drug Use and Health, 1999-2001, la N ational Health Interview Survey, 1978-2001, la Current Population Survey, 1998-1999, las N ational Vital Statistics, 1999, y el U.S. C ensus Bureau, 2001. Resultados. En Estados Unidos de América se ha visto una reducción en la prevalencia del uso de cigarillos, tanto en los jóvenes co mo en los adultos. Como grupo se ha observado una reducción entre quienes indicaron en las encuestas realizadas ser mexicanos o mexico-americanos (ambos grupos combinados). Los mexicanos o mexico-americanos jóvenes y adultos presentan un consumo de cigarrillos menor que los blancos no hispanos o los indios americanos. Sin embargo, esta tasa menor entre los mexicanos y mexico-americanos se explica mayormente por la menor tasa de consumo de cigarillos entre las mujeres mexicanas y mexico-americanas (combinadas). Esto es, mientras que las mujeres mexicano americanas y mexicanas (combinadas) presentan una prevalencia menor de consumo de cigarrillos que las blancas no hispanas, los hombres mexicano americanos y mexicanos (com-

\author{
Caraballo RS, Lee CW. \\ Tobacco use among Mexicans \\ and their descendants in the United States. \\ Salud Publica Mex 2004;46:241-250. \\ The English version of this paper \\ is available at: http://www.insp.mx/salud/index.html
}

\begin{abstract}
A bstract
Objective. To show the information obtained in U.S. surveys and studies on cigarette smoking or other tobacco use in Mexicans residing in the United States. Material and Methods D ifferent information systems and surveys were used.Those used in the study herein presented include the Youth Risk Behavior Survey, 1991-2001, the N ational Survey on D rug U se and Health, 1999-2001, the $\mathrm{N}$ ational $\mathrm{Health}$ Interview Survey, 1978-2001, the Current Population Survey, 1998-1999, The N ational Health Vital Statistics, 1999, and the U.S. Census Bureau, 2001. Results. A decreased prevalence of cigarette smoking has been observed in the U.S. both in young persons and adults. A decreased prevalence among subjects reporting Mexican and Mexican-A merican (combined) ethnicity was also noted.Young adults and adults of Mexican or Mexican-A merican origin smoke cigarettes less frequently than non-Hispanic whites or A merican Indians. However, this lower rate among Mexicans and Mexican-Americans is due mainly to the lower use of cigarettes among M exican-A merican and Mexican women (combined). Although these women have a lower prevalence of cigarette smoking than non-Hispanic white females, among Mexican-A merican and Mexican males (combined) cigarette smoking may be as common as in non-H ispanic white males. Moreover, those who identify themselves as Mexican-A merican have higher cigarette use than those who identify themselves as Mexicans. Finally, Mexican and Mexican-American women (combined) of a lower education level are more prone to smoking during pregnancy than females of the same
\end{abstract}

(1) N ational Center for Chronic Disease Prevention and Health Promotion, Centers for Disease Control and Prevention, Atlanta, GA. USA.

(2) Epidemiologic Intelligence Service, Epidemiology Program 0 ffice, Centers for Disease Control and Prevention, Atlanta, GA. USA.

Fecha de recibido: 11 de mayo de 2004 • Fecha de aprobado: 16 de junio de 2004

Solicitud de sobretiros: Dr. Ralph S Caraballo. Mail Stop K-50, 0 ffice on Smoking and Health, National Center for Chronic Disease Prevention and Health Promotion, Centers for Disease Control and Prevention, 4770 Buford Highway, N .E., Atlanta, USA, GA 30341-3724.

Correo electrónico: E-mail: rfc8@ cdc.gov 
binados) posiblemente fuman tanto como los blancos no hispanos. En adición, en general, quienes se identifican a sí mismos como mexicano-americanos presentan mayores tasas de consumo de cigarrillos que aquellos que se autoidentifican como mexicanos. Por último, las mujeres mexicanas y mexicano-americanas (combinadas) de un nivel educativo menor son más dadas a fumar durante el embarazo que las mexicanas y mexicano-americanas con una escolaridad más elevada. Conclusiones. Este reporte demuestra diferencias por edad, género, autodefinición (mexicano o mexicano-americano), y nivel educativo en el hábito de fumar entre los mexicanos o descendientes de mexicanos que viven en Estados U nidos deA mérica. Es esencial comprender los cambios demo gráficos y los patrones y tendencias de consumo de tabaco entre los mexicanos y mexicano-americanos en este país, para poder diseñar e implementar programas de control del tabaquismo que sean eficaces, culturalmente apropiados y diseñados específicamente para los mexicanos y mexicano-americanos. El texto completo en inglés de este artículo está disponible en: http:/ /www.insp.mx/salud/index.html

Palabras clave: tabaco; fumar; mexicanos; mexicano-americanos; jóvenes; adultos; ho mbres; mujeres; Estados Unidos de América group with a higher education level. Conclusions This report shows differences by age, sex, self-definition of ethnicity (Mexican or Mexican-A merican), and education level, regarding smoking among Mexicans or persons with a M exican background living in the United States. It is crucial to under stand the demo graphic changes and trends and patterns among Mexicans and Mexican-A mericans in the U.S. so as to design and implement smoking control programs that are efficient, culturally sensitive, and designed specifically for Mexicans and Mexican-A mericans. The English version of this paper is available at: http://www.insp.mx/salud/ index.html

Key words: to bacco, smoking, Mexicans, Mexican-A mericans, young adults, adults, males, females, U nited States of A merica
$\mathrm{E}$ n Estados Unidos de América (EUA), el consumo de tabaco es la primera causa de muerte prevenible. ${ }^{1}$ Se estima que 45.8 millones de adultos fuman cigarrillos, ${ }^{2}$ provocando más de 440000 muertes y más de 75 mil millones de dólares en gastos médicos directos por año. ${ }^{3}$ Cada año, 8.6 millones de personas se enferman gravemente y se pierden 5.6 millones de años de vida potencial debido al tabaquismo. ${ }^{3}$ En este artículo presentamos temas relacionados con el tabaquismo entre jóvenes y adultos mexicanos y descendientes de mexicanos que viven en EUA.

La cantidad de personas descendientes de mexicanos que residen en EUA (mexicano-americanos*) se ha incrementado enormemente durante las últimas dos décadas. En 1980, había 8.7 millones de mexicano-americanos viviendo en EUA, y en el año 2000 la cifra llegó a 20 millones. ${ }^{4}$ Esto representa un incremento de $136 \%$ en 20 años. Los 20.6 millones de personas dentro de este país que en el año 2000 se identificaron a sí mismos como mexicanos o de origen mexicano representan $7.3 \%$ de su población, y

\footnotetext{
* En este reporte definimos como mexicano-americanos a las personas que se identificaron a sí mismos como mexicanos o como mexicano-americanos en diferentes encuestas o registros estadísticos vitales.
}

$58.5 \%$ de todos los "hispano/latinos". Si la proporción de mexicano-americanos entre los hispanos de EUA se mantiene en ese nivel, a mediados de 2004 habrá 21.6 millones de mexicano-americanos y 57.5 millones en $2050{ }^{4}$

La mayoría de los mexicano-americanos viven sólo en unos cuantos estados de EUA. ${ }^{4}$ Hasta el año 2000, dos terceras partes de todos los mexicano-americanos vivían en California o en Texas. California contaba con $41 \%$ (8.5 millones) del total de mexicano-americanos de todo el país y Texas con 25\%, o sea, 5.1 millones. Otro $15 \%$ vivía en Illinois, Arizona, Colorado y Florida. Esto indica que en el año 2000 más de $80 \%$ de todos los mexicano-americanos residían en estos seis estados. Durante la última década, a pesar de que la cantidad de hispanos que vivían en Carolina del Norte, Georgia, Tennessee, Arkansas, Carolina del Sur y Alabama era mucho menor que en California y Texas, estos estados también registraron una elevada tasa de crecimiento de la población hispana, incluyendo a muchos mexicanoamericanos (Carolina del Norte aumentó de 655\%*;

\footnotetext{
* Los números entre paréntesis representan el porcentaje de aumento de la población de mexicano-americanos entre 1990 y 2000.
} 
Georgia, 460\%; Tennessee, 458\%; Arkansas, 390\%; Carolina del Sur, 379\%; Alabama 368\%). ${ }^{4}$

Es esencial comprender la cambiante composición general de la población de EUA debido a los especiales retos relacionados con la salud que se pueden derivar de esos cambios. La comprensión de los cambios demográficos en el país y los patrones y tendencias de consumo de tabaco entre los mexicano-americanos es especialmente importante porque nos puede ayudar a diseñar e implementar programas eficaces de control del tabaquismo específicamente destinados a los mexicano-americanos.

\section{Jóvenes}

\section{Consumo de cigarrillos}

La iniciación en el consumo de tabaco se presenta fundamentalmente en los primeros años de la adolescencia, una etapa del desarrollo alejada por varias décadas de la muerte y la discapacidad asociadas al consumo de tabaco en la edad adulta. ${ }^{5}$ Mientras más pronto empiezan los jóvenes a fumar cigarrillos, es más probable que lo sigan haciendo una vez que se vuelven adultos; es menos probable que lo dejen y el tiempo en que serán consumidores es potencialmente más largo, incrementando de esta manera la posibilidad de desarrollar enfermedades crónicas relacionadas con el tabaquismo. ${ }^{5}$ La adicción a la nicotina asegura que muchos adolescentes consumidores de tabaco lo seguirán siendo al convertirse en adultos.

En EUA, no es sino hasta muy recientemente cuando se dispone de datos sobre consumo de tabaco entre los jóvenes mexicano-americanos. Si bien, las encuestas suelen preguntar por la identidad racial/étnica de los entrevistados, en la mayoría de los casos la cantidad de éstos es demasiado pequeña para poder extraer conclusiones significativas para determinados grupos específicos, incluyendo a los mexicano-americanos. Sin embargo, los estudios llevados a cabo entre jóvenes hispanos en general pueden ilustrar información relevante sobre los patrones y conductas asociados al consumo de tabaco de los jóvenes mexicano-americanos, dado que éstos representan $69 \%$ de todos los hispanos que tienen entre 10 y 19 años de edad. ${ }^{6}$

En esta sección daremos información obtenida en dos Encuestas Nacionales basadas en las escuelas que brindan datos sobre el consumo de tabaco entre los hispanos en general. Posteriormente, daremos información más específica proveniente de mexicano-americanos que participaron en una encuesta de hogares a escala nacional.

Históricamente, las encuestas basadas en las escuelas de EUA han arrojado estimaciones de una más alta prevalencia de consumo de tabaco que las encuestas de hogares. Estas estimaciones pueden diferir por varias razones. Entre las diferencias más importantes están las que se refieren a los periodos de recolección de la información, a las preguntas específicas que se formulan, al contexto en el cual se recoge la información, a los métodos usados para manejar asuntos relacionados con la privacidad e, incluso, a factores del azar.

La Encuesta de Comportamientos de Riesgo entre los Jóvenes, ${ }^{7}$ de 2001, realizada entre estudiantes de preparatoria (grados 9 a 12) indicó que $28.5 \%$ de todos los estudiantes de preparatoria de EUA eran fumadores actuales de cigarrillos. Se definió a los fumadores actuales como aquellos que habían fumado uno o más días durante los 30 días anteriores a la encuesta. La prevalencia de fumadores actuales de cigarrillos fue de $26.6 \%$ para los estudiantes hispanos, $31.9 \%$ para los blancos no hispanos y $14.7 \%$ para los negros no hispanos (figura 1). Para estos tres grupos, la prevalencia de consumo de cigarrillos aumentó* entre 1991 y 1997. Sin embargo, entre 1997 y 2001, la prevalencia en el consumo se redujo para los tres grupos.

La Encuesta Nacional de Tabaquismo entre los Jóvenes ${ }^{8}$ mostró que la prevalencia global de fumado-

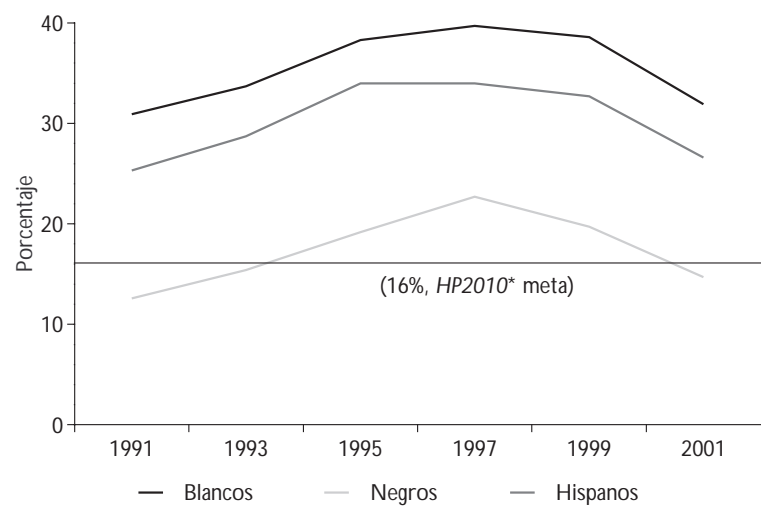

* Healthy people (gente saludable) 2010

Figura 1. Porcentaje de estudiantes de preparatoria QUE REPORTARON HABER FUMADO CIGARRILLOS RECIENTEMENTE, SEgún RAZA/ETnia, Estados Unidos de AmÉricA, 1991-2001

\footnotetext{
* A menos que se anote por separado, los términos "incremento", "decremento", "más alto" y "más bajo" se usan para describir cambios en estimaciones o comparaciones entre grupos sólo si el cambio o la diferencia son estadísticamente significativos en el intervalo de confianza (IC) de 95\% .
} 
res entre los estudiantes de secundaria (grados 6 a 8) era de $10.1 \% .{ }^{9}$ Las estimaciones de consumo de cigarrillos no variaron de manera significativa, según los distintos grupos raciales o étnicos. Los estudiantes hispanos de secundaria tenían una prevalencia de tabaquismo de $11.4 \%$, seguidos por los negros no hispanos $(11.2 \%)$ y los blancos no hispanos (10.8\%).

Una Encuesta Nacional de EUA sobre Consumo de Drogas y Salud ${ }^{10}$ (NSDUH por sus siglas en inglés, 1999-2001, datos combinados) ofrece información adicional sobre el consumo de cigarrillos entre jóvenes de catorce grupos raciales o étnicos diferentes. Entre los mexicano-americanos de 12 a 17 años de edad, $11.0 \%$ reportaron haber fumado cigarrillos durante el mes previo a la encuesta. Esta tasa no es diferente para la totalidad de la población hispana $(11.8 \%)$, pero es menor que la prevalencia de consumo de tabaco en ese grupo de edad para todos los grupos raciales/étnicos (13.8\%). Tanto para los hombres como para las adolescentes los mexicano-americanos mostraron una menor prevalencia de consumo de cigarrillos que los blancos no hispanos y los indios americanos o los nativos de Alaska, pero una prevalencia mayor que los negros y los asiáticos (figura 2). Las tasas de consumo de tabaco no variaron significativamente entre los adolescentes mexicanos y todos los hispanos, según el sexo.

En la misma encuesta, la edad promedio de los mexicano-americanos para haber fumado parte de o

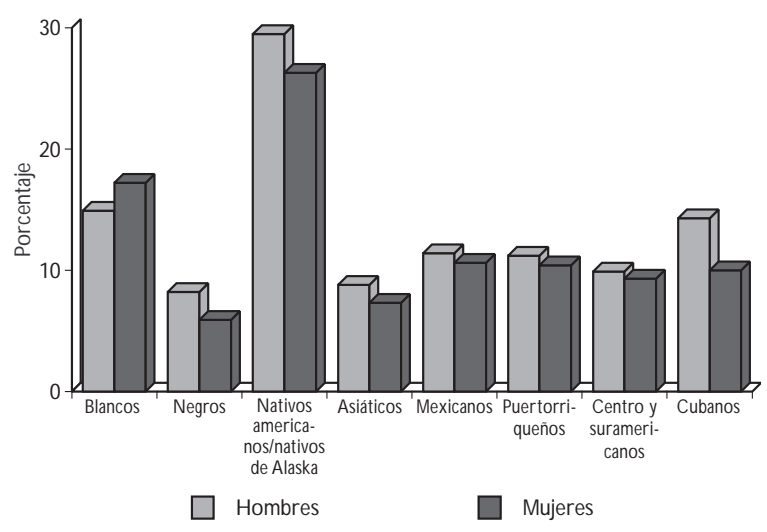

Fuente: N ational Survey on Drug-Use and Health, 1999, 2000, 2001, datos combinados.

Figura 2. Consumo de cigarrillos durante el últiMO MES ENTRE JÓVENES (12 A 17 AÑOS DE EDAD) POR SEXo y RAZA/EtNIA, Estados Unidos de AmérICA, 1999 2001 todo un cigarrillo por primera vez fue de 15.9 años y la edad promedio para el consumo diario de cigarrillos fue de 18.7 años, casi tres años más tarde. Cerca de 3 de cada 10 menores mexicano-americanos no fumadores eran susceptibles de empezar a fumar. Con base en las preguntas hechas en la misma encuesta ( $i \mathrm{Si}$ uno de tus mejores amigos te ofrece un cigarrillo, te lo fumarías? y ¿Crees que en algún momento dentro de los próximos 12 meses te fumarás un cigarrillo?) se calculó que el entrevistado no era susceptible de empezar a fumar si a ambas preguntas respondió "definitivamente no". Se consideró que cualquier otra combinación de "definitivamente sí", "tal vez sí", "tal vez no" o "definitivamente no" daba como resultado "susceptible de empezar a fumar". Los jóvenes mexicano-americanos parecieron ser más susceptibles a empezar a fumar que otros grupos raciales o étnicos a excepción de los indios americanos y los nativos de Alaska, los centro y suramericanos y los cubanos (cuadro I).

Por lo que se refiere a la preferencia de marcas de cigarrillos, más de 6 de cada 10 fumadores mexicanoamericanos actuales entre los 12 y los 17 años de edad (65.2\%) fumaban Marlboro, comparados con $70.2 \%$ de los jóvenes asiáticos, $60.3 \%$ de los blancos, $60.1 \%$ de los indios americanos y los nativos de Alaska y 7.2\% de los negros. Entre los jóvenes fumadores negros, Newport $(77.5 \%)$ fue la marca preferida de cigarrillos, mientras que alrededor de 2 de cada $10(17.2 \%)$ de los jóvenes mexicano-americanos fumaban Newport.

\section{Cuadro I \\ SUSCEPTIBILIDAD DE EMPEZAR A FUMAR ENTRE LOS JÓVENES NO FUMADORES DE ESTADOS UNIDOS DE AMÉrICA (12 A 17 AÑos DE EDAD), 1999-2001}

\begin{tabular}{|c|c|c|}
\hline Raza/etnia & $\begin{array}{c}\text { \% Susceptible } \\
\text { de empezar } \\
\text { a fumar }\end{array}$ & $\begin{array}{c}\text { Intervalo } \\
\text { de confianza } \\
\text { de } 95 \%\end{array}$ \\
\hline \multicolumn{3}{|l|}{ o-hispanos } \\
\hline Blancos & 23.0 & $22.4-23.6$ \\
\hline N egros/africano-americanos & 27.1 & $25.8-28.5$ \\
\hline Indios americanos/nativos de Alaska & 27.9 & $21.6-35.2$ \\
\hline Asiáticos & 20.4 & $18.1-23.0$ \\
\hline
\end{tabular}

\begin{tabular}{lll}
$\begin{array}{l}\text { Hispanos } \\
\text { Mexicanos }\end{array}$ & 31.5 & $30.1-33.0$ \\
\hline Puertorriqueños & 22.6 & $19.4-26.2$ \\
\hline Centro o Suramericanos & 27.9 & $24.3-31.8$ \\
\hline Cubanos & 27.1 & $20.0-35.5$
\end{tabular}

Fuente: N ational Household Survey on Drug Abuse, 1999, 2000, 2001, datos combinados

salud pública de méxico / vol.46, no.3, mayo-junio de 2004 
Los porcentajes de jóvenes que habían fumado con anterioridad, pero no durante el último mes son de $22.0 \%$ para los mexicano-americanos y $21.3 \%$ para todos los grupos raciales/étnicos combinados. Entre los hombres adolescentes, la proporción de mexicanoamericanos que fumaron con anterioridad, pero no en el último mes, fue de $24.0 \%$, comparado con $22.3 \%$ de los hispanos y $22.8 \%$ de los blancos no hispanos. En el caso de las adolescentes, $19.9 \%$ de las mexicanas reportaron haber fumado anteriormente, mientras que $20.2 \%$ del total de las hispanas y $21.6 \%$ de las blancas no hispanas dijeron haber fumado antes, pero no durante el último mes. No se presentaron diferencias significativas entre estos grupos raciales/étnicos.

\section{Uso de otros productos de tabaco}

Los cigarrillos no son el único producto de tabaco usado por los jóvenes que viven en EUA. De acuerdo con la U.S. National Youth Tobacco Survey de 2002, ${ }^{9}$ entre los estudiantes hispanos de secundaria, los cigarros puros son el segundo producto de tabaco más popular luego de los cigarrillos (prevalencia de 6.3\%), seguidos por la pipa $(4.4 \%)$, los bidis* $(2.9 \%)$, el tabaco sin humo $(2.9 \%)$ y los kreteks $\ddagger(2.6 \%)$. Después del consumo de cigarrillos, los estudiantes hispanos de preparatoria usaron puros con mayor frecuencia $(10.8 \%)$, luego pipa $(4.6 \%)$, tabaco sin humo (3.8\%), bidis (3.5\%) y kreteks (3.0\%).

Factores asociados con la iniciación a fumar cigarrillos entre los mexicano-americanos

Los factores asociados con la iniciación en el consumo de cigarrillos pueden variar de acuerdo con los grupos raciales o étnicos; ${ }^{11}$ sin embargo, algunos factores de riesgo que predicen la iniciación pueden ser comunes a todos los adolescentes más que únicos para los de alguno de los grupos. Algunos de esos factores comunes son la presión de los pares, de los hermanos, de los padres o de quienes los cuidan para que empiecen a fumar, el comportamiento de fumadores entre amigos, hermanos o padres/cuidadores, la edad del niño/a, problemas de conducta del niño/a, y su receptividad a la publicidad y promoción del tabaco. ${ }^{5,11-18}$

Otros factores parecen ser más específicos o que afectan de manera distinta a los grupos raciales o étnicos, como el precio de los cigarrillos, o la aculturación de los jóvenes, la actitud hacia el consumo de tabaco y

\footnotetext{
* Cigarrillos con diferentes aromas originarios de la India (n. del T.).

‡ Cigarrillos con clavo de olor originarios de Indonesia (n. del T.).
}

los beneficios percibidos por fumar. ${ }^{11,19-23}$ Un estudio de los factores de riesgo asociados con el consumo de cigarrillos entre los jóvenes mexicano-americanos sugiere que la elevada aceptación de normas culturales mexicanas (esto es, la baja aculturación dentro de la cultura anglo de EUA) juega un papel protector. ${ }^{24}$

\section{Adultos}

\section{Consumo de cigarrillos}

El consumo de tabaco está ligado a varios problemas crónicos de salud que afectan a todos los grupos de población una vez que llegan a la edad adulta. Entre ellos se encuentran las enfermedades cardiovasculares, el cáncer y las enfermedades respiratorias agudas. Las enfermedades relacionadas con el consumo de tabaco imponen una pesada carga evitable sobre las poblaciones adultas, al mismo tiempo que una importante presión económica en términos de gastos médicos directos y costos indirectos, en términos de pérdida de productividad. El problema del consumo de tabaco entre los mexicano-americanos está amplificado dado que algunos de ellos tienen menor acceso que los blancos no hispanos a información anti-tabaco cultural y lingüísticamente apropiada, a materiales educativos, mensajes en los medios de comunicación y servicios para dejar de fumar. Además, los mexicano-americanos, al igual que otras minorías, han sido -y siguen siendo- blanco de los esfuerzos publicitarios de la industria del tabaco, incluyendo el patrocinio de eventos culturales, el financiamiento de organizaciones culturales y otro tipo de campañas de acercamiento. ${ }^{18}$ De este modo, la prevención del consumo de tabaco entre los mexicano-americanos es un paso crítico en la eliminación de esa epidemia en EUA. La fortaleza y ventajas específicas de las comunidades mexicanoamericanas implican una importante plataforma para lanzar iniciativas eficaces y culturalmente relevantes de control del consumo de tabaco.

La encuesta NSDUH (datos combinados 19992000) mostró que $22.8 \%$ de los adultos mexicano-americanos de 18 años de edad y más reportó haber fumado durante los últimos 30 días, mientras que la prevalencia general de EUA fue de $26.5 \% .{ }^{12}$ La prevalencia reportada para los mexicano-americanos fue menor que la de los blancos no hispanos, la de los negros no hispanos, la de los indios americanos o nativos de Alaska y la de los puertorriqueños, pero más alta que la de los asiático-americanos (cuadro II). Otros estudios también han demostrado una menor prevalencia de consumo actual entre los mexicano-americanos que entre los blancos no hispanos de EUA..$^{25,26}$ 
Datos históricos muestran que en las dos últimas décadas la prevalencia del consumo de cigarrillos entre los mexicano-americanos ha decrecido gradualmente (National Health Interview Survey, 1978-2001, figura 3). Datos de la National Health Interview Survey (NHIS) (la cual define el consumo actual de tabaco como "haber fumado por lo menos 100 cigarrillos y actualmente fumar todos los días o en algunos días", en vez de "haber fumado durante los últimos 30 días", como en la NSDUH), también indican que la prevalencia de consumo de cigarrillos de los mexicano-americanos ha decrecido de $29.8 \%$, en 1978 , a $16.1 \%$ en 2001 (-13.7 puntos porcentuales).

Esta tendencia descendente fue general entre los mexicano-americanos, los blancos no hispanos y los negros no hispanos (figura 3). No obstante, la magnitud del decrecimiento en el consumo de cigarrillos entre los mexicano-americanos durante los últimos 23 años fue más alto (-13.7 puntos porcentuales) que entre los otros blancos no hispanos (-10.0 puntos porcentuales).

Cuando se incluyen otros productos de tabaco como tabaco para mascar, rapé,* puros y tabaco para pipa, la estimación de prevalencia entre los adultos de 18 años de edad y más en EUA que reportaron haber consumido alguna forma de tabaco en los últimos 30 días es de $31.3 \%$. Esta misma estimación es de $24.6 \%$ entre los mexicano-americanos (NSDUH, 1999-2001, datos combinados).

Considerando el género, la prevalencia de consumo actual de cigarrillos fue más alta para los hombres que para las mujeres en todos los grupos raciales/étnicos, salvo para los indios americanos/nativos de Alaska (figura 4). En el caso de los mexicano-americanos, los hombres (29.8\%) tienen el doble de la tendencia a fumar que las mujeres (15.6\%). Los estudios han demostrado que las mujeres mexicano-americanas tienen una prevalencia menor de consumo de tabaco que otras mujeres blancas no hispanas, mientras que la prevalencia para los hombres es similar en ambos grupos. ${ }^{25}$ Durante los años 80, no obstante, los hombres mexicano-americanos mostraron un modesto descenso en su tasa de consumo de cigarrillos, pero no se detectó la misma tendencia entre las mujeres. ${ }^{27}$ Por otra parte, los estudios han mostrado que las mujeres mexicanas que viven en EUA presentan una tasa de consumo de cigarrillos mucho más alta que sus contrapartes que viven en México. ${ }^{27,28}$

\footnotetext{
* Tabaco picado para inspirar (n. del T.)
}

\section{Cuadro II \\ Consumo de cigarrillos en el mes previo ENTRE ADULTOS POR RAZA Y ETNIA. Estados Unidos de AmÉrica, 1999 a 2001}

$\begin{array}{ccc} & \text { Consumo de } & \text { Intervalo } \\ \text { cigarrillo en el mes } & \text { de confianza } \\ \text { anterior (\%) } & \text { de } 95 \%\end{array}$

\begin{tabular}{lll}
$\begin{array}{l}\text { No-Hispanos } \\
\text { Blancos }\end{array}$ & 27.4 & $26.9-27.9$ \\
\hline N egros/africano-americanos & 25.7 & $24.4-27.0$ \\
\hline Indios americanos/nativos de Alaska & 40.4 & $35.2-45.8$ \\
\hline A siáticos & 16.2 & $14.1-18.6$
\end{tabular}

Hispanos

\begin{tabular}{lll} 
Mexicanos & 22.8 & $21.4-24.4$ \\
\hline Puertorriqueños & 30.4 & $26.5-34.7$ \\
\hline Centro o Suramericanos & 21.3 & $18.5-24.5$ \\
\hline Cubanos & 19.2 & $16.0-22.8$
\end{tabular}

Fuente:U.S.C urrent N ational Survey on D rug U se and Health, 1999, 2000, 2001, datos combinados

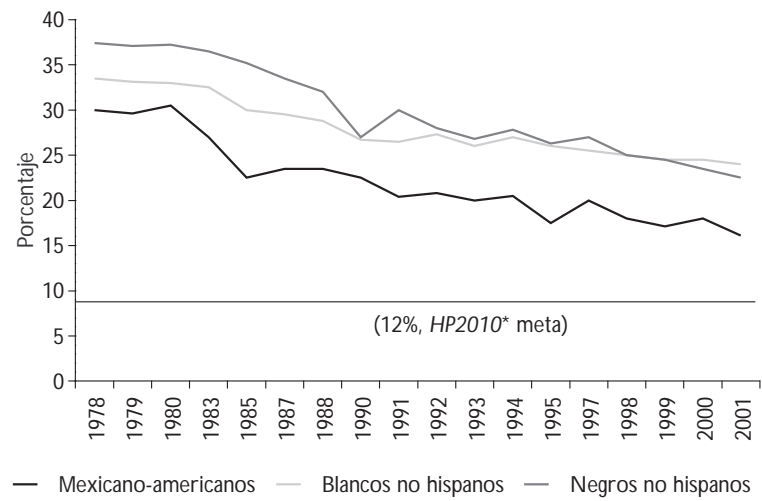

* Health people (gente saludable) 2010

₹ Para los datos de 1997 a 2001 los mexicano-americanos incluyen a las personas que se identifican a sí mismas como mexicanos o como mexicano-americanos

Fuente: N ational Health Interview Survey, 1978-2001

Figura 3.Tendencias en porcentaje de consumo actUAL DE CIGARRILlos ENTRE ADULtos de Estados UNIDOS DE AMÉRICA DE 18 AÑOS DE EDAD O MÁS, POR RAZA/ ETNIA, 1978-2001 ${ }^{\ddagger}$ 


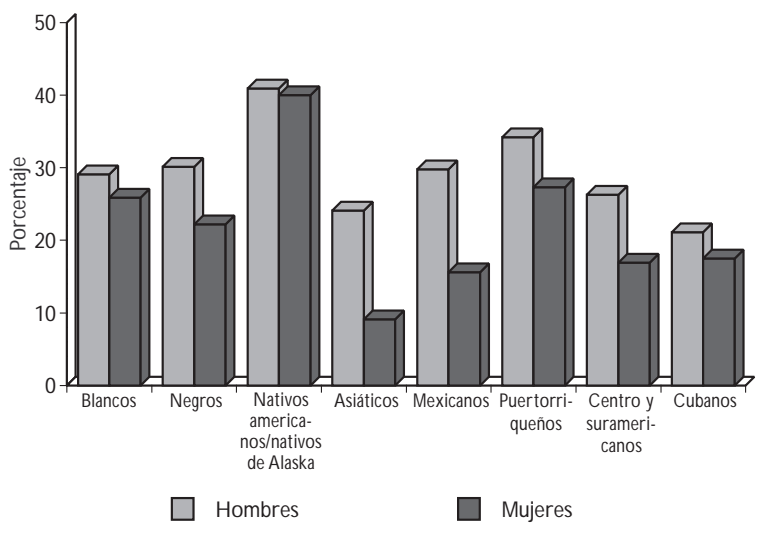

Fuente: N ational Survey on D rug U se and Health, 1999-2001

Figura 4. Consumo de cigarrillos durante el ÚltiMO MES ENTRE ADULTOS (18 AÑOS DE EDAD O MÁS), POR SEXo y RAZa/etNIA, Estados Unidos de América, 19992001

A partir de 1997, la National Health Interview Survey de EUA ha preguntado a los entrevistados de origen mexicano si se identifican a sí mismos como mexicanos o como mexicano-americanos. La figura 5 presenta la prevalencia de consumo de cigarrillos entre la gente de origen mexicano de acuerdo con su autoidentificación étnica. De 1997 a 2000, los mexicano-americanos presentaron una prevalencia ligeramente más alta que quienes se identificaron a sí mismos como mexicanos. Sin embargo, en 2001, no se ve ninguna diferencia en la prevalencia de consumo de cigarrillos entre los dos grupos (mexicano-americanos 16.3\%; mexicanos 16.0\%). La significancia estadística de las diferencias no fue analizado.

La figura 6 resume la prevalencia del consumo de cigarrillos de los mexicano-americanos y los mexicanos de acuerdo con su residencia geográfica. Tal como se dijo, la mayoría de los mexicano-americanos viven en California, Texas, Illinois, Arizona, Colorado y Florida. En lo que se refiere a los mexicano-americanos que viven en California y Texas, donde se localiza 2/3 parte de esa población, las prevalencias de consumo actual de cigarrillos fueron de $14 \%$ (mexicano-americanos) y 13.8\% (mexicanos) en California y $20.4 \%$ (mexicano-americanos) y $13.8 \%$ (mexicanos) en Texas. La figura 6 también demuestra que las personas que se identifican a sí mismas como mexicanoamericanos tienen tasas más altas que los que se identifican como mexicanos en la mayoría de los estados, salvo en Colorado y Florida (datos para mexi-

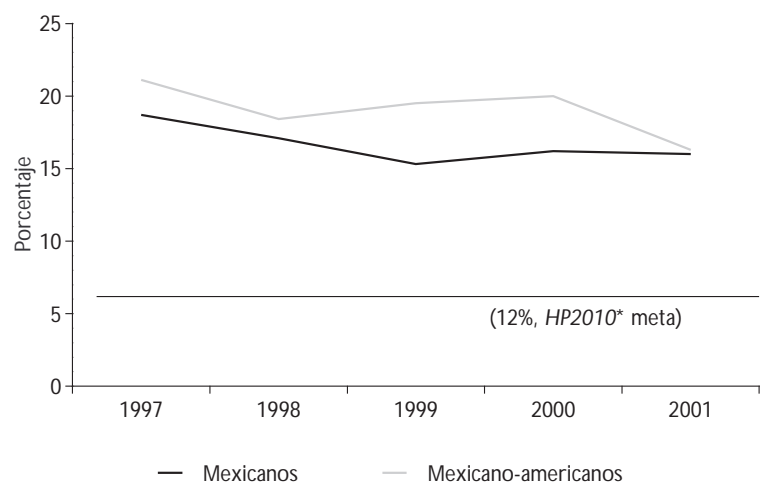

* Health people (gente saludable) 2010

Figura 5. Prevalencia de consumo de cigarrillos, SEgún aUtoidentificación, Estados Unidos de AmÉRICA, 1997-2001

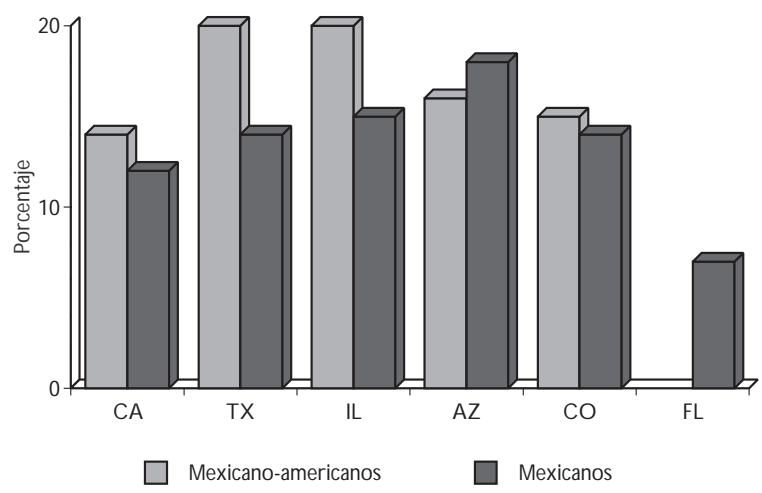

Fuente: Current Population Survey, 1998-1999, datos combinados

Figura 6. Prevalencia de consumo actual de cigaRRILLOS POR ESTADOS Y AUTO-IDENTIFICACIÓN, ESTADOS Unidos de AmÉRICA, 1998-1999

cano-americanos en Florida no disponibles debido al reducido tamaño de los grupos). La significancia estadística de las diferencias no fue examinado.

$\mathrm{Al}$ analizar la prevalencia del consumo de cigarrillos en diferentes grupos de edad, los mexicano-americanos de 55 años de edad y más presentaron la prevalencia estimada más baja $(13.4 \%)$. Los adultos mexicano-americanos entre los 18 y los 34 años de edad tuvieron una prevalencia estimada de $17.1 \%$ y los de 35 a 54, de 18.3\% (NHIS, 1999-2001, datos combinados).

Varios estudios han indicado que los fumadores mexicano-americanos fuman menos cigarrillos al día que 
otros grupos raciales/étnicos. ${ }^{25,29,30}$ La NHIS (1997-2001, datos combinados) mostró que $89.4 \%$ de los fumadores mexicano-americanos fuman menos de 15 cigarrillos al día, $8.6 \%$ fuman un promedio de 15 a 24 al día y $2.0 \%$ fuman más de 24 al día. Esto se puede comparar con las cifras de los fumadores blancos no hispanos: $70.0 \%, 20.6 \%$ y $9.4 \%$, respectivamente. Entre los fumadores negros no hispanos la proporción de los que fuman menos de 15 cigarrillos al día fue de $79.3 \%$, mientras que $17.3 \%$ fuman 15 a 24 al día y 3.4\% fuman más de 24 .

Datos nacionales anteriores (NHIS, datos combinados para 1987, 1988, 1990 y 1991) demostraron que $68 \%$ de los mexicano-americanos fumaban menos de 15 cigarrillos al día, mientras que todos los grupos no hispanos tenían una tasa de $32 \%$. Sólo $6 \%$ de los fumadores mexicano-americanos eran considerados como grandes fumadores (24 o más cigarrillos al día) mientras que la tasa de los grupos no hispanos era de $25 \%$. El U.S. Surgeon General's Report on Tobacco Use Among U.S. Racial/Ethnic Minority Groups reportó que 65\% de los fumadores hispanos de EUA fumaban menos de 15 cigarrillos al día, 27.3\% fumaban de 15 a 24 y $7.7 \%$ fumaban más de $24 .{ }^{18}$ La comparación entre estos datos con los más recientes del NHIS demuestra que la proporción de grandes fumadores decreció entre los fumadores mexicano-americanos de $6 \%$ a $2 \%$, mientras que la de los que fuman menos de 15 cigarrillos al día aumentó de $68 \%$ a $89 \%$.

Los estudios también han descubierto que los fumadores mexicano-americanos tienden más a tratar de dejar de fumar que los fumadores blancos no hispanos. Usando datos de una encuesta telefónica del Community Intervention Trial for Smoking Cessation (COMMIT), Hymowitz y colaboradores ${ }^{30}$ descubrieron que los mexicano-americanos tienden más a haber intentado de dejar de fumar al menos una vez que los otros grupos raciales/étnicos (cociente de probabilidades=1.17; intervalo de confianza de 95\%: 1.05-1.30).

Otros datos nacionales disponibles muestran que cerca de $44 \%$ de los mexicano-americanos que eran fumadores, ya no lo son. Entre los antiguos fumadores (aquellos que habían fumado más de 100 cigarrillos a lo largo de su vida) que se identificaron a sí mismos como mexicano-americanos, $44.4 \%$ reportaron haber dejado de fumar; y entre los que se auto-identificaron como mexicanos, $44.2 \%$ dijeron que ya no fumaban. Se tienen datos disponibles sobre la proporción de personas que alguna vez fumaron en su vida (100 cigarillos o mas)* y que de-

\footnotetext{
* Las definiciones de "alguna vez fumó" hablan tanto de fumadores que han fumado más de 100 cigarrillos a lo largo de su vida, como de aquellos que ya no fuman (n. del T.).
}

jaron de fumar en los estados en los que reside la mayoría de los mexicano-americanos. La figura 7 presenta esa información para los mexicano-americanos, de acuerdo con su localización geográfica. La proporción de personas que alguna vez fumaron en su vida y que dejaron de fumar varía mucho, según los estados, tanto para los mexicano-americanos como para los mexicanos.

Una encuesta nacional (NSDUH, 1999-2001, datos combinados) preguntó a los adultos de 18 años y más a qué edad fumaron su primer cigarrillo. Los resultados demostraron que la edad promedio en la que se fumó por primera vez es menor entre los mexicanos (15.9 años) que entre los negros (16.4 años) y los asiáticos (16.8 años). Aunque la diferencia no es estadísticamente significativa, la edad en la que los fumadores mexicano-americanos comienzan a fumar es ligeramente menor que entre la totalidad de la población hispana (16.6 años). Esta diferencia entre los mexicano-americanos y la totalidad de los hispanos está explicada en gran medida por el comportamiento de las mujeres en relación con el consumo de tabaco. Mientras que la edad en la que los hombres mexicano-americanos suelen empezar a fumar (15.6 años) es similar a la de todos los hispanos (15.7 años), las mujeres mexicano-americanas fuman su primer cigarrillo antes (16.2 años) que el resto de las mujeres hispanas (17.5 años). Por otra parte, aunque los hombres mexicano-americanos fuman su primer cigarrillo antes que las mujeres de su grupo, ambos empiezan el consumo diario casi a la misma edad (hombres: 18.9 años; mujeres: 18.6 años).

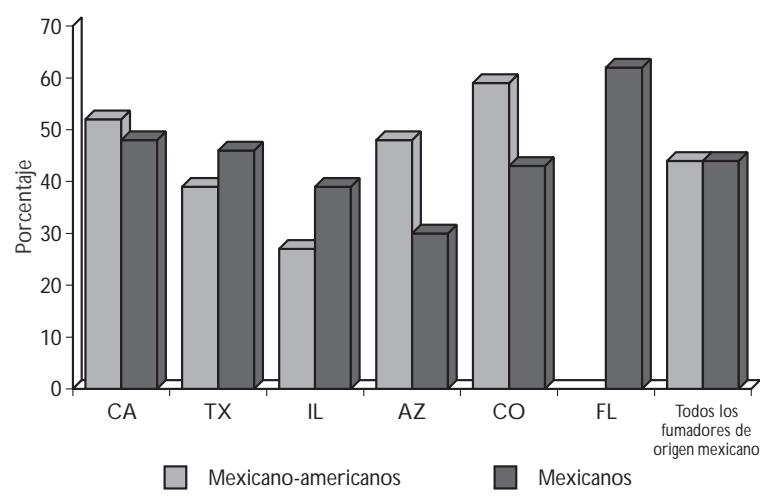

Figura 7. Porcentaje de personas que alguna vez FUMARON Y QUE HAN DEJADO DE FUMAR, POR ESTADO Y autoidentificación. Estados Unidos de AmÉRica, 1998-1999 


\section{Mujeres embarazadas}

Los efectos perniciosos del consumo de tabaco durante el embarazo son ampliamente conocidos. Sin embargo, alrededor de $12 \%$ de las mujeres de EUA siguen fumando cigarrillos durante el embarazo, ${ }^{31}$ aunque la prevalencia varía según los grupos raciales/étnicos. Los datos nacionales de los certificados de nacimiento de EUA para 1999 muestran que las mujeres hispanas en general tienen una menor prevalencia de consumo de tabaco durante el embarazo (3.7\%) que las indias americanas o las nativas de Alaska (20\%), que las blancas no hispanas (15.7\%), o que las negras no hispanas $(9.1 \%){ }^{32}$ En el caso de las mujeres hispanas el consumo de cigarrillos durante el embarazo decreció de 6.7\% en 1990 a 3.2\% en 2001. ${ }^{31,32}$ Entre las mujeres mexicano-americanas la prevalencia fue incluso más baja en 2001, situándose en $2.4 \% .^{31}$

Los estudios han descubierto que las tasas de consumo de cigarrillos durante el embarazo son más bajas para las mujeres con 16 o más años de educación que para las que tenían una escolaridad de 9 a 11 años. ${ }^{33}$ Los datos nacionales de certificados de nacimiento de 1999 mostraron que entre las mujeres embarazadas mexicano-americanas al decrecer el nivel educativo se incrementan las tasas de consumo de tabaco (figura 8). Las intervenciones destinadas a promover que las mujeres embarazadas dejen de fumar deben determinar cómo llegar mejor a las que tienen un nivel educativo más bajo.

\section{Conclusiones}

Tanto para adultos como para adolescentes, la prevalencia de consumo de cigarrillos reportada de 1999 a 2001 fue menor entre los mexicano-americanos que entre los blancos no hispanos o entre los indios americanos/nativos de Alaska, pero más alta que entre los asiáticos (NSDUH).

Entre los adolescentes mexicano-americanos entre los 12 y los 17 años de edad, $11.0 \%$ reportaron ser fumadores actuales (NSDUH, 1999-2001, datos combinados). La estimación del consumo de cigarrillos entre los jóvenes mexicano-americanos es ligeramente más baja que la tasa de los jóvenes de todos los grupos raciales/étnicos combinados. Alrededor de $9.8 \%$ de los jóvenes mexicano-americanos también reportaron haber usado otros productos de tabaco tales como bidis, puros, kreteks o tabaco sin humo (NSDUH).

La prevalencia de consumo de cigarrillos entre los adultos mexicano-americanos declinó gradualmente ente 1978 y 2001, pasando de $29.8 \%$ a 16.1\% (NHS). Las personas que se identifican a sí mismas como mexi-

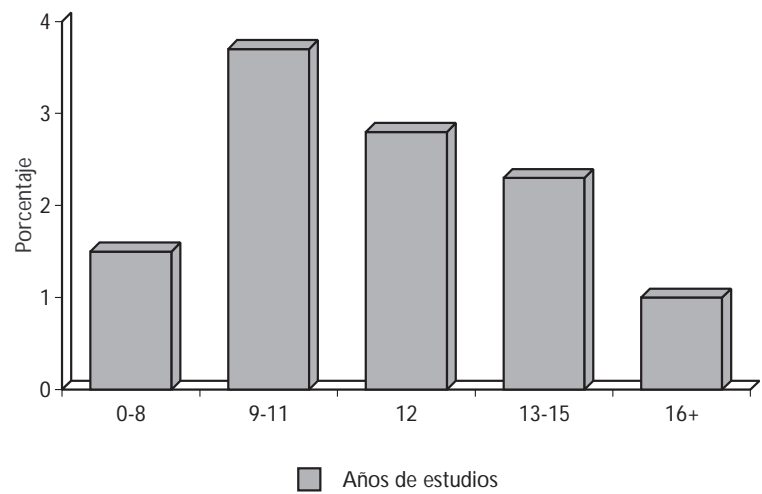

Fuente: N ational Vital Statistics Reports,Vol 49, No 7; agosto 28, 2001

Figura 8. Prevalencia de consumo de tabaco duranTE EL EMBARAZO ENTRE MUJERES MEXICANO-AMERICANAS Según su Nivel educativo, Estados Unidos de AmÉRICA, 1999

cano-americanos presentan mayores tasas de consumo de cigarrillos que quienes se autoidentifican como mexicanos. Casi una cuarta parte $(24.6 \%)$ de los adultos mexicano-americanos reportó haber usado algún tipo de producto de tabaco en los últimos 30 días (NHIS). Los adultos mexicano-americanos reportaron fumar menos cigarrillos por día que las personas de otros grupos raciales/étnicos; más de $89 \%$ de los adultos fumadores mexicano-americanos reportaron fumar menos de 15 cigarrillos al día, mientras que la tasa para los blancos no hispanos es de 70\% (NHIS).

Son más los adultos hombres que las mujeres mexicano-americanos que fuman cigarrillos. Mientras que las mexicano-americanas presentan una prevalencia menor de consumo de cigarrillos que las mujeres blancas no hispanas, los hombres mexicano-americanos fuman tanto como los blancos no hispanos (NSDUH). La prevalencia de consumo de cigarrillos durante el embarazo entre las mujeres mexicano-americanas es más alta entre aquellas que tienen un menor nivel educativo.

\section{Referencias}

1. McG innis JM, Foege W H. Actual causes of death in the United States. JAMA 1993;270:2207-2212.

2. Centers for Disease Control and Prevention. Cigarette smoking among adults-United States, 2002. MMW R Morb Mortal W kly Rep 2004;53(20):427-431.

3. Centers for Disease Control and Prevention. Annual smokingattributable mortality, years of potential life lost, and economic costs- 
United States, 1995-1999. MMW R Morb Mortal W kly Rep 2002;51(14):300-303.

4. U.S. Census Bureau. Census 2000 Summary File 1 U nited States. W ashington, DC: US Census Bureau; 2001. Disponible en: http:// www.census.gos/Press-Release/www/2001/sumfile.introd 5. U.S. D epartment of Health and Human Services. Preventing tobacco use among young people:A Report of the Surgeon General. 1994 Atlanta (GA): US D epartment of Health and Human Services, Public Health Service, $C$ enters for D isease Control and Prevention, $N$ ational Center for Chronic Disease Prevention and Health Promotion, 0 ffice on Smoking and Health; 1994.

6. Current Population Survey, datafiles. Disponible en: http:// www.bls.census.gov/cps/cpsmain.htm. [2001 mayo 4].

7.Youth Risk Behavior Surveillance System, Data \& Statistics. Disponible en: http://www.cdc.gov/H ealthyYouth/yrbs/index.htm. [2004 mayo 4]. 8. American Legacy Foundation. D isponible en: http:// www.americanlegacy.org/americanlegacy/skins/alf/ display.aspx?Action=display_page $\&$ mode $=U$ ser $\&$ Modulel $D=8 \mathrm{cde} 2 \mathrm{e} 88$ 3052-448c-893d-d0b4b14b31c4\&0 bjectID =391985a0-198d-41e1-a6ec0e40fa351287. [2004 mayo 12].

9. Centers for Disease Control and Prevention. Tobacco use among middle and high school students-United States, 2002. MMW R Morb Mortal W kly Rep 2003;52(45):1096-1098.

10. Centers for Disease Control and Prevention. Prevalence of cigarette use among 14 racial/ethnic populations-U nited States, 1999-2001. MMW R Morb Mortal W kly Rep 2004;53(3):49-52.

11. Griesler PC, Kandel D B, D avies M. Ethnic differences in predictors of initiation and persistence of adolescent cigarette smoking in the $\mathrm{N}$ ational Longitudinal Survey of Youth. N icotine Tob Res 2002;4(1):79-93. 12. US D epartment of Health and Human Services and $N$ ational Institute of $\mathrm{H}$ ealth, $\mathrm{N}$ ational $\mathrm{C}$ ancer Institute. Changing adolescent smoking prevalence. Atlanta (GA): USD H HS; 2001. (Monograph 14). 13. Ling PM, G lantz SA.W hy and how the tobacco industry sells cigarettes to young adults: evidence from industry documents. Am J Public Health 2002;92(6):908-16.

14. Sargent JD, Beach ML, D alton MA, Mott LA, Tickle JJ, A hrens MB et al. Effect of seeing tobacco use in films on trying smoking among adolescents: C ross sectional study. Br Med J 2001;323(7326):1394-1397. 15. Alexander CS,Allen P, Crawford MA, McC ormick LK. Taking a first puff: Cigarette smoking experiences among ethnically diverse adolescents. Ethnic Health 1999;4:245-257.

16. Everett $S A, W$ arren CW, Sharp D, Kann L, Husten C G, Crossett LS. Initiation of cigarette smoking and subsequent smoking behavior among US high school students. Prev Med 1999;29:327-333.

17. Siegel M, Biener L, Rigotti N A. Effect of tobacco sales laws on adolescent smoking initiation. Prev Med 1999;29:334-342.

18. U.S. Department of Health and Human Services. Tobacco use among U.S. racial/ethnic minority groups-A frican A mericans, A merican
Indians and A laska natives, A sian A mericans and Pacific Islanders, and Hispanics: A Report of the Surgeon General. 1998. Atlanta (GA): US Department of Health and Human Services, Public Health Service, Centers for D isease Control and Prevention, $N$ ational $C$ enter for Chronic Disease Prevention and Health Promotion, 0 ffice on Smoking and Health; 1998.

19. Kaplan CP, N apoles-Springer A, Stewart SL, Pérez-Stable EJ. Smoking acquisition among adolescents and young Latinas:The role of socioenvironmental and personal factors. Addict Behav 2001;26:531-550. 20. Anderson C, Burns D M. Patterns of adolescent smoking initiation rates by ethnicity and sex. Tob Control 2000;9(Suppl 2):ii4-ii8. 21. DeC icca P, Kenkel D, Mathios A. Racial differences in the determinants of smoking onset.J Risk Uncertainty 2000;1:311-340. 22. Chen $X$, Unger JB, Johnson CA. Is acculturation a risk factor for early smoking initiation among $C$ hinese A merican minors? Tob Control 1999;8:402-410.

23. Kegler MC, Kingsley B, Malcoe LH, Cleaver V, Reid J. Functional value of smoking and nonsmoking from the perspective of American Indian youth. Fam Community Health 1999;22:31-42.

24. Morgan-Lopez AA, Castro FG, Chassin L, MacKinnon D P.A medicated moderation model of cigarette use among Mexican American youth. Addict Behav 2003;28:583-589.

25. C oreil J, Ray LA, Markides KS. Predictors of smoking among Mexican Americans: findings from the Hispanic HAN ES. Prev Med 1991;20:508-517. 26. Giovino GA, Schooley MW, Z hu BP, Chrismon JH, Tomar SL, Peddicord JP et al. Surveillance for selected tobacco-use behaviorsUnited States, 1900-1994. CDC Surveillance Summaries. MMW R Morb Mortal W kly Rep 1994;43:SS-3.

27. Escobedo LG Remington PL. Birth cohort analysis of prevalence of cigarette smoking among $\mathrm{H}$ ispanics in the U nited States. JAMA 1989;261:66-68.

28. Pletsch PK. Prevalence of cigarette smoking in Hispanic women of childbearing age. N urs Res 1991;40:103-105.

29. Caraballo RS, Giovino GA, Pechacek TF, Mowery PD, Richter PA, Strauss W J et al. Racial and ethnic differences in serum cotinine levels of cigarette smokers:Third $\mathrm{N}$ ational $\mathrm{H}$ ealth and $\mathrm{N}$ utrition Examination Survey, 1988-1991. JAMA 1998;280(2):135-139.

30. Hymowitz N, Corle D, Royce J, Hartwell T, Corbett K, O rlandi M, Piland N. Smokers' baseline characteristics in the COMMIT trial. Prev Med 1995;24:503-508:30.

31. Martin JA, Hamilton BE,Ventura SJ, Menacker F, Park MM, Sutton PD. Births: Final data for 2001. N atl Vital Stat Rep 2002;51(2).

32. Mathews TK. Smoking during pregnancy in the 1990s. N atl Vital Stat Rep 2001;49(7).

33. US D epartment of Health and Human Services. W omen and smoking:A report of the Surgeon General.W ashington, DC: Public Health Service, 0 ffice of the Surgeon General; 2001. 